\title{
Magic Angle Spinning NMR Metabolomics
}

\section{Jian Zhi Hu*}

Biological Science Division, Pacific Northwest National Laboratory, WA 99354, USA

\section{Editorial}

Nuclear Magnetic Resonance (NMR) spectroscopy is a nondestructive, quantitative, reproducible, untargeted and unbiased method that requires no or minimal sample preparation, and is one of the leading analytical tools for metabolomics research [1-3]. The easy quantification and the no need of prior knowledge about compounds present in a sample associated with NMR are advantageous over other techniques $[1,4] .{ }^{1} \mathrm{H}$ NMR is especially attractive because protons are present in virtually all metabolites and its NMR sensitivity is high, enabling the simultaneous identification and monitoring of a wide range of low molecular weight metabolites.

However, the resolution of the ${ }^{1} \mathrm{H}$ NMR spectra from intact tissues is often poor due to the unwanted line broadenings arising from isotropic magnetic susceptibility variations near boundaries of inter- and intracellular structures, residual homo-nuclear proton dipolar coupling and residual chemical shift anisotropy interaction. It is well-known that all of these line broadenings can be eliminated by the technique of Magic Angle Sample Spinning (MAS), where the rotor containing the sample is rotating rapidly about an axis that is inclined at an angle of $54.7356^{\circ}$ with respect to the external main magnetic field [5]. The technique, termed as high-resolution MAS (i.e., ${ }^{1} \mathrm{H}$ hr-MAS) [6] using a sample spinning rate of several $\mathrm{kHz}$ or more, generates a high resolution ${ }^{1} \mathrm{H}$ NMR metabolite spectrum of intact biological tissue samples with spectral resolution approaching that obtained from standard liquid state NMR on cell and tissue extracts. The unique ability of hr-MAS to study intact tissue samples, i.e., the elimination of extraction process, is a major breakthrough in metabolomics, not only because the extraction takes time and also there is always a concern that some metabolites might be lost during an extraction process. During the last decade, there have been numerous publications in which ${ }^{1} \mathrm{H}$ hr-MAS has been successfully used to study a variety of biochemical processes associated with disease progression and/or the effects of therapies etc. [7].

Typically about 10-60 mg of tissue samples are used for metabolic profiling using the technique of hr-MAS. Recently, a breakthrough has been made in miniaturizing the hr-MAS technique using the concept of Magic Angle Coil Spinning (MACS) [8,9]. MACS uses wireless inductive coupling between the static coil that is used for signal excitation and reception, and a tuned micro-coil that is co-rotating with the sample container. Because the micro-coil is wound directly on capillary sample tube, the sample filling factor is high, resulting in a high sensitivity. MACS is capable of metabolic profiling on tissue sample with mass as small as $0.2 \mathrm{mg}$. Despite its remarkable success, MACS has the following limitations. (i) A micro-coil (and a fixed capacitor attached to the coil) has to be wound on every sample tube that inevitably increases the cost of the experiment. (ii) MACS favors extremely small sample tube detection by using extremely small diameter of copper wires for winding the micro RF coil due to the factor that the eddy current from a very small rotating micro $\mathrm{RF}$ coil is minimized so that high resolution can be obtained. The drawback is that it is not easy to load a tissue sample into a, e.g., $400 \mu \mathrm{m}$ inner diameter sample tube, thus limiting the throughput of the experiment.

Hr-MAS has now been widely accepted as a powerfulNMR metabolic profiling techniques on intact biological tissues samples, and has made indispensable contributions to the success of the rapidly developing field of metabolomics. However, despite its success in metabolomics, hr-MAS is technically challenging due to the use of a sample spinning rate of several $\mathrm{kHz}$ or more. These challenges include: (i) Tissue samples contain a significant amount of fluids, mostly $\mathrm{H}_{2} \mathrm{O}$, accounting for more than $90 \%$ of the tissue mass. Because quantification is vitally important for NMR metabolomics plus the safety concerns arising from HIV and other potentially infectious disease, a leakage free sealing system at high spinning rate is required for generating quantitative results and for protecting both the experimenters and the expensive MAS probe. Unfortunately, production of a $100 \%$ leakage free sample rotor for $\mathrm{hr}$ MAS is challenging due to the large centrifugal force associated with fast sample spinning, especially when the same sample rotor is used repeatedly. (ii) The large centrifugal force associated with fast sample spinning of several $\mathrm{kHz}$ or more is destructive to the tissue structure and even some of the cells [10]. As a result, the same sample cannot be used for further analysis by other techniques such as histopathology after hr-MAS. (iii) For large sized biological tissues of $\sim 300 \mathrm{mg}$ or more, it is even more difficult to spin the sample to several $\mathrm{kHz}$. Studying a single organ from a laboratory animal, or larger sample from surgery room can be very important for toxicology assessment and/or disease diagnosis. The application of hr-MAS would be difficult in these cases.

To overcome the technical challenges associated with hr-MAS, my group has recently developed a new approach, called slow-MAS NMR [11-21] that significantly enhances the spectral resolution in excised tissues, organs and live small animals. With one of the methods that we developed based on an existing solid state NMR method originally reported by Antzutkin et al. [22], namely ${ }^{1} \mathrm{H}$ PASS (Phase-Adjusted Spinning Sidebands) $[11,12]$, sample spinning rates as low as $40 \mathrm{~Hz}$ can be employed. The method of ${ }^{1} \mathrm{H}$ PASS facilitates non-destructive ex vivo studies of excised intact tissues and organs. ${ }^{1} \mathrm{H}$ PASS requires relatively short (from a few minutes to less than an hour) measurement times and offers both high sensitivity and high spectral resolution. Note that a sample spinning rates up to $600 \mathrm{~Hz}$ have been reported safe for maintaining the cellular structure of excised prostate tissue [23]. Furthermore, it is easy to keep the fluids inside the tissue without fluid leakage at slow sample spinning rates. Importantly, we have carefully compared the spectral resolution obtained from both the $1 \mathrm{H}$ slow-MAS at a spinning rate of $80 \mathrm{~Hz}$ and $1 \mathrm{H} \mathrm{hr}-\mathrm{MAS}$ at a spin rate of $2 \mathrm{kHz}$ by using a sample consisting of an intact left lung lobe from a mouse by performing slow-MAS experiment first, followed immediately by hrMAS on the same sample. We found [20] that the spectral resolution from slow-MAS is significantly higher than that from hr-MAS primarily due to the severe redistribution of the tissue mass at high speed, thus

*Corresponding author: Jian Zhi Hu, Biological Science Division, Pacific Northwest National Laboratory, WA 99354, USA, Tel: 5093716544; E-mail: Jianzhi.Hu@pnnl.gov

Received May 24, 2016; Accepted May 25, 2016; Published May 31, 2016

Citation: Hu JZ (2016) Magic Angle Spinning NMR Metabolomics. Metabolomics 6: e147. doi:10.4172/2153-0769.1000e147

Copyright: (c) $2016 \mathrm{Hu} \mathrm{JZ}$. This is an open-access article distributed under the terms of the Creative Commons Attribution License, which permits unrestricted use, distribution, and reproduction in any medium, provided the original author and source are credited. 
the creation of magnetic susceptibility gradient along the magic angle axis that cannot be eliminated by MAS.

We expect that with further development the technology of slow-MAS NMR metabolomics will play a vital role in studying the biochemistry non-destructively in intact biological tissues with variable sample sizes ranging from as small as less than $1 \mu \mathrm{l}$ to as large as $1 \mathrm{~cm}^{3}$ or more using a single probe. The small sample volume detection is important for sample limited applications where high spatial sampling for a single type of cells in tissue is required, or where minimally invasive biopsy detection in a laboratory animal or a patient is needed. The large sample volume detection on biological samples with size of $1.0 \mathrm{~cm}^{3}$ or more is useful for investigating an entire intact organ from a small laboratory animal or tissues from a surgery room in a clinical setting. This will be important for investigating whole organ injury due to a variety kind of insults in laboratory animals, or disease diagnosis in patients.

\section{References}

1. Larive CK, Barding GA Jr, Dinges MM (2015) NMR Spectroscopy for Metabolomics and Metabolic Profiling. Anal Chem 87: 133-146.

2. Lindon JC, Nicholson JK, Holmes E, Everett JR (2000) Metabonomics: Metabolic processes studies by NMR spectroscopy of biofluids. Concepts Magn Reson 12: 289-320.

3. Lindon JC, Holmes E, Nicholson JK (2004) Toxicological applications of magnetic resonance. Progress in Nuclear Magnetic Resonance Spectroscopy 45: 109-143

4. Bollard ME, Stanley EG, Lindon JC, Nicholson JK, Holmes E (2005) NMRbased metabonomic approaches for evaluating physiological influences on biofluid composition. NMR in Biomedicine 18: 143-162.

5. Bollard ME, Garrod S, Holmes E, Lindon JC, Humpfer E, et al. (2000) Highresolution ${ }^{1} \mathrm{H}$ and ${ }^{1} \mathrm{H}-{ }^{13} \mathrm{C}$ Magic Angle Spinning NMR Spectroscopy of Rat Liver. Magn Reson Med 44: 201-207.

6. Cheng LL, Ma MJ, Becerra L, Ptak T, Tracey I, et al. (1997) Quantitative neuropathology by high resolution magic angle spinning proton magnetic resonance spectroscopy. Proc Natl Acad Sci USA 94: 6408-6413.

7. Beckonert O, Coen M, Keun HC, Wang Y, Ebbels TMD, et al. (2010) Highresolution magic-angle-spinning NMR spectroscopy for metabolic profiling of intact tissues. Nature Protocols 5: 1019-1032.

8. Sakellariou D, Goff GL, Jacquinot JF (2007) High-resolution, high-sensitivity NMR of nanolitre anisotropic samples by coil spinning. Nature 447: 694-698.

9. Wong A, Li X, Sakellariou D (2013) Refined Magic-Angle Coil Spinning Resonator for Nanoliter NMR Spectroscopy: Enhanced Spectral Resolution. Anal Chem 85: 2021-2026.
10. Weybright P, Millis K, Campbell N, Cory DG, Singer S (1998) Gradient high-resolution, magic angle spinning ${ }^{1} \mathrm{H}$ nuclear magnetic resonance spectroscopy of intact cells. Magn Reson Med 39: 337-345

11. Wind RA, Hu JZ, Rommereim DN (2001) High Resolution ${ }^{1} \mathrm{H}$ NMR Spectroscopy in Organs and Tissues Using Slow Magic Angle Spinning Magn Reson Med 46: 213-218.

12. Wind RA, Hu JZ (2006) In vivo and ex vivo high-resolution ${ }^{1} \mathrm{H}$ NMR in biological systems using low-speed magic angle spinning. Progress in Nuclear Magnetic Resonance Spectroscopy 49: 207-259.

13. Hu JZ, Rommerein DN, Wind RA (2002) High resolution ${ }^{1} \mathrm{H}$ NMR spectroscopy in rat liver using magic angle turning at a $1 \mathrm{~Hz}$ spinning rate. Magn Reson Med 47: 829-836.

14. Wind RA, Hu JZ, Rommereim DN (2003) High-resolution 1H NMR spectroscopy in a live mouse subjected to $1.5 \mathrm{~Hz}$ magic angle spinning. Magn Reson Med 50: 1113-1119.

15. Hu JZ, Wind RA (2002) The evaluation of different slow-MAS techniques at low spinning rates in aqueous samples and in the presence of magnetic susceptibility gradient. J Magn Reson 159: 92-100.

16. Hu JZ and Wind RA (2003) Sensitivity-enhanced phase corrected ultra-slow magic angle turning using multiple-echo data acquisition. J Magn Reson 163: $149-162$

17. Hu JZ, Wind RA, Rommereim DN (2006) ${ }^{1} \mathrm{H}$ relaxation times of metabolites in biological samples obtained with non-destructive ex vivo slow-MAS NMR. Magn Reson Chem 44: 269-275.

18. Hu JZ, Wind RA, Mclean J, Gorby YA, Resch CT, et al. (2004) Highresolution ${ }^{1} \mathrm{H}$ NMR spectroscopy of metabolically active microordanisms using non-destructive magic angle spinning. Spectroscopy 19: 98-102.

19. Wind RA, Hu JZ, Majors PD (2006) Localized in vivo isotropic-anisotropic correlation ${ }^{1} \mathrm{H}$ NMR spectroscopy using ultraslow magic angle spinning. Magn Reson Med 55: 41-49.

20. Hu JZ, Rommereim DN, Minard KR, Woodstock A, Harrer BJ, et al. (2008) Metabolomics in lung inflammation: a high resolution ${ }^{1} \mathrm{H}$ NMR study of mice exposed to silica dust. Toxicology Mechanisms and Methods 18: 1-14.

21. Hu JZ (2011) Slow Magic Angle Sample Spinning: A Non- or Minimally Invasive Method for High- Resolution 1H Nuclear Magnetic Resonance (NMR) Metabolic Profiling. Methods in Molecular Biology 708: 335-364

22. Antzutkin ON, Shekar SC, Levitt MH (1995) Two-dimensional sideband separation in magic-angle-spinning NMR. J Magn Reson 115: 7-19.

23. Taylor JL, Wu CL, Cory D, Gonzalez RG, Bielecki A, et al. (2003) Highresolution magic angle spinning proton NMR analysis of human prostate tissue with slow spinning rates. Magn Reson Med 50: 627-632. 